\title{
Are Regular Capacity Building Courses Formative? Perspective of School Directors and Educators
}

DOI: https://doi.org/10.32870/dse.v0i12.256

Ana Cecilia Valencia Aguirre*

Resumen: La presente investigación surge de la necesidad de comprender cómo impactan los cursos de formación continua que ofrecen las instancias formadoras de docentes en la práctica cotidiana y la subjetividad de los actores que han recibido este tipo de formación. Este aspecto es analizado desde la opinión de la propia comunidad escolar y de los directores que han sido formados en dichos cursos.

Se parte de concebir la formación como un trayecto que le brinda al sujeto un sentido de comunidad en el oficio y de identidad en la acción. El propósito es valorar las percepciones de directores sobre su capacitación y conocer si ésta contribuye a su formación desde la valoración que nos ofrece la comunidad docente conformada por veintitrés escuelas básicas de educación primaria que concluyeron en el año 2013 un curso de formación continua (SEJ, 2006). Palabras clave: formación, directores, capacitación.

Abstract: This article arises from the need to visualize the impact of regular capacity building courses offered by the different educator forming organizations in the everyday practice and the subjectivity of the actors who have received this kind of formation. The analysis is based on the opinion of the members of the school community itself and the objective is toThe point of departure is the idea of formation as a journey which gives the subject a sense community in the profession and of identity in action. Keywords: capacity building, formation as community, identity.

\section{Nuevas demandas, nuevos retos de formación}

Los directivos escolares fueron considerados a partir de los años noventa del siglo veinte, actores estratégicos en la transformación escolar y en el logro de la calidad educativa, ya que se inicia una serie de cambios sociales propiciados por el advenimiento de un estado evaluador ante la crisis del modelo benefactor heredado de las economías de la posguerra de los años cuarenta y, en el caso mexicano, de la postrevolución mexicana del siglo veinte, así como del proceso de descentralización administrativa, económica y el arribo de nuevas formas de ciudadanía y democracia participativa.

${ }^{1}$ Doctora en Educación. Profesora investigadora en el Departamento de Filosofía de la Universidad de Guadalajara. Especialista en la línea: Sujetos y actores de la educación. Correo electrónico: anaval_a@hotmail.com 
Lo anterior implicó nuevos imperativos a la tarea docente, a la gestión de las escuelas llevada a cabo por los directores escolares, a los propósitos de la escolarización, a los sistemas educativos y a las políticas educativas en su conjunto (Braslavsky y Acosta, 2001).

Desde esa década se dio la necesidad de lograr la calidad educativa centrada en mejorar los resultados educacionales, bajar la tasa de reprobación, deserción y rezago educativo y lograr una mayor equidad en la distribución de la oferta educativa entre distintos grupos sociales. Dichas acciones obedecieron entre otras cosas a las tendencias de las economías de mercado que demandaban mayor competitividad en el contexto de la globalización y a las nuevas exigencias de formación del capital humano. En este escenario se inician procesos reformistas en diversos países de América Latina y México no es la excepción.

Ante estos procesos de reformas educativas y de reforma de los sistemas educativos ${ }^{1}$ quedó clara la tesis de que no es posible lograr esos cambios necesarios si no se parte del corazón de cambio que es la escuela.

Los antecedentes de esta tesis son los aportes de las escuelas eficaces, movimiento iniciado en Inglaterra que afirma que la escuela puede obtener buenos logros si se accede a una comunidad de aprendizaje desde los actores, donde se involucre la sociedad y el contexto desde lógicas centradas en aprendizajes relevantes. Dicho movimiento centra la atención en la dirección de las escuelas como clave para el logro de los aprendizajes relevantes o aprendizajes para la vida; porque de la gestión de un director de escuela depende que se den los cambios esperados o bien que se frenen las posibilidades de mejora escolar, como señala Schmelkes:

Ni duda cabe que una buena escuela empieza por un buen director, ya que el directivo adquiere una relevancia que quizá antes se había ignorado. En manos del director, de hecho, se encuentra el poder -o bien de obstaculizar- [para] que se dé un proceso comprometido (Schmelkes, 1998: 190).

De ahí que consolidar al directivo como una autoridad con liderazgo resulte una de las intenciones fundamentales de la formación bajo el modelo reformista, cuyo propósito es impulsar el desarrollo de competencias para que éstos sean capaces de implementar procesos de formación permanente en el logro de la calidad desde las escuelas pues, como señala Zorrilla y Pérez: "Al director le corresponde realizar el tejido fino que permita unir la dimensión pedagógica con la gestión escolar" (Zorrilla y Pérez, 2006: 115).

Desde esta perspectiva se generan cambios en las estructuras económicas, políticas y culturales que conllevarían a nuevas demandas y exigencias educativas y a distintas formas de inter-

\footnotetext{
${ }^{1}$ Hay una diferencia esencial entre ambos conceptos: el primero refiere a la renovación del currículo y los enfoques pedagógicos, el segundo se centra en los cambios de las lógicas de la gestión y la administración educativa en su conjunto desde el particular (Zorrilla y Pérez, 2006).
} 
pelación del trabajo docente y de la dirección escolar, dado que las nuevas tareas directivas ya no se limitarían a responder a las lógicas de un modelo de administración centralista, sino a crear condiciones en la escuela para generar aprendizajes de calidad, fortalecer los proyectos de mejora escolar desde la participación comunitaria y movilizar a distintas agencias y actores estratégicos articulados en el logro de las metas escolares desde procesos comunicativos y de transparencia (Zorrilla y Pérez, 2006).

Se parte de la necesidad de formar a los directivos y supervisores escolares, situación que se reconoce a través de los diagnósticos tanto nacionales como internacionales, que señalan:

Existe una necesidad de formación que puede denominarse básica para la gestión y la política educativa que debe alcanzar a todos los actores que intervienen en educación. Esa formación básica consiste en el desarrollo de ciertas competencias que, por otra parte, es conveniente que desarrollen todos los ciudadanos: la detección de necesidades; la concertación de alianzas; la negociación de conflictos; la anticipación de problemas; el planteo de alternativas a partir de un sólido conocimiento de las existentes y de los debates que existen en torno a ellas; la discusión de las alternativas entre los actores involucrados, su puesta en práctica y seguimiento, y la rendición de cuentas públicas respecto de los resultados (Braslavsky y Acosta, 2001: 14).

En el caso concreto de México esta tendencia inicia a partir de la década de los años noventa con el advenimiento de tres documentos fundacionales de la política educativa que son el Acuerdo Nacional para la Modernización de la Educación Básica (1992), la Ley General de Educación (1993) y el Programa de Desarrollo Educativo (1995-2000); éstos generan nuevas demandas de formación de directivos e implementación de programas de formación continua destinados a promover las competencias exigidas en el naciente campo de la gestión escolar, lo que habrá de generar una nueva ruta en los procesos formativos de directores escolares y supervisores.

En este sentido, Aguilar y Schmelkes señalan que:

La necesidad de conocimiento ante los cambios acusados y las nuevas funciones emergentes del cargo constituyen otra vía que condujo a sectores de personal directivo hacia programas de formación. En este contexto surge la oferta académica en gestión, que... se concentra en las instituciones formadoras del magisterio de educación básica y normal (Aguilar y Scmelkes, 2001: 204). ${ }^{2}$

En el contexto de estos nuevos escenarios se va generando una oferta que exige una formación que responda a estos desafíos. De ahí que una prioridad de la política educativa sea el que las secretarías de educación de los estados desarrollen proyectos de formación tendientes a formar compe-

\footnotetext{
${ }^{2}$ Los puntos suspensivos son propios.
} 
tencias de gestión en los directivos con la finalidad de responder a las exigencias de las tendencias reformistas, ${ }^{3}$ en el caso concreto del estado de Jalisco, se implementó en el 2006 un proyecto de formación de directivos basado en un modelo por competencias, el Programa de Formación de Directivos por Competencias (PFDC, 2006, en delante), ${ }^{4}$ que reconoce como parte su diagnóstico la necesidad de formar a los directores ya que éstos han llegado al cargo sin una formación específica que les permita el desempeño de sus nuevas funciones, en el mejor de los casos han arribado al nombramiento pasando del aula donde fungieron como profesores a una dirección escolar que les exige el manejo de nuevas competencias propias de la función:

Hasta ahora el Sistema Educativo Estatal ha aceptado que la sola formación inicial como profesor de grupo y el ascenso al puesto sean elementos suficientes para que el director cuente con el perfil y las competencias necesarias para desarrollar una tarea tan compleja (SEJ/PFDC, 2006:3-4).

Esta perspectiva estatal coincide con el diagnóstico del Instituto Internacional de Planeamiento de la Educación (IIPE) que señala:

De ahí que desarrollar procesos formativos que incrementen o coadyuven la capacidad de gestión de los actores educativos sea cuestión de no poca importancia. El acceso a cargos directivos de diversa jerarquía tanto en el sistema de educación básica como en el de educación media superior y superior, se da fundamentalmente mediante una carrera al interior de cada estructura, fincada de modo parcial o total en relaciones políticas o político-académicas. Es decir, los estudios y las certificaciones académicas no son un criterio definitorio en el acceso a los cargos directivos (Aguilar y Schmelkes, en Braslavsky y Acosta, 2001: 269).

Las competencias a desarrollar en los directivos y que demandarían una formación específica para este profesional, de acuerdo con Braslavsky y Acosta, serían: "la gestión estratégica, el liderazgo, la comunicación, la delegación, la negociación, la resolución de problemas, la anticipación, el trabajo en equipo, el discernimiento ético" (Braslavsky y Acosta, 2001: 15).

Estas competencias responden a la necesidad de fortalecer en los directores la capacidad de gestión, esto es, de reconvertir un modelo centralista fundado en la administración y planeación educativa hacia un ejercicio cotidiano que coloca en el centro a la escuela y provee a través de proyectos estratégicos planes de mejora desde la participación comunitaria. Por ello, se demandan programas formativos que:

\footnotetext{
${ }^{3}$ En el diagnóstico elaborado por el IIPE (2001), Aguilar y Schmelkes consideran que la oferta de formación en el campo de la gestión en México abarcó en el año 2000, 111 programas de licenciatura, posgrado y diplomados que ofrecían instituciones que se dividían en tres ámbitos: el normativo, el de las universidades e instituciones públicas de educación superior y el de las universidades e instituciones de educación superior privadas. Cada uno de estos ámbitos atiende a poblaciones específicas, que se insertan en nichos también específicos.

${ }^{4}$ Programa que inició en el año 2006 y concluyó en el año 2014. Como todo proyecto, quedó trunco ante la discontinuidad de los responsables de los proyectos de formación, de la experiencia que implicó una asignación de recursos públicos para su operación se preserva la presente evaluación realizada en el 2012 y de la cual se siguen infiriendo interesantes análisis como el presente.
} 
Fortalezcan la capacidad de los actores educativos de diagnosticar la situación local (escuela, zona escolar, sector educativo, municipio, estado, región, país, etc.) en favor de una visión democrática, y en función de ello diseñar, realizar y evaluar proyectos. Asimismo, para favorecer una visión democrática es necesario indagar en el significado local que dicha visión puede tener. Conocer las redes institucionales, los mecanismos locales de gestión y las tradiciones en las formas de relación institucionales permite tener una visión del horizonte real y posible de participación de los actores, así como de las vías para incentivarla (Aguilar y Schmelkes, en Braslavsky y Acosta, 2001: 269).

De acuerdo a este diagnóstico elaborado desde instancias internacionales, en el país se inicia desde la década de los años noventa el crecimiento en la oferta curricular para la formación continua en el ámbito de la gestión. Sin embargo, a pesar de la recurrencia de las instancias oficiales, de las universidades tanto públicas como privadas de formar en este ámbito, poco conocimiento se tiene de cómo impactan o interpelan estos modelos de formación a los actores que la reciben, lo que implicaría conocer cómo desde las condiciones situacionales o contextuales del trabajo cotidiano los actores encuentran significaciones que le atribuyan nuevos sentidos a su práctica. Tampoco se sabe qué condiciones permiten lograr las transferencias del nivel discursivo al nivel práctico en caso de que éstas se concretaran, ni cómo influyen otros factores del contexto situacional o personal de los profesionales en dichos procesos.

\section{La formación desde los sujetos}

La formación es ante todo un proceso de subjetivación, donde el sujeto en interacción se forma a sí mismo (Anzaldúa, 2009). Sin embargo, el sujeto está frecuentemente olvidado, como lo señala Ducoing:

Ese sujeto frecuentemente olvidado en todo aquello que de él es inasible, incierto, incontrolable e impredecible; ese sujeto cuya formación le ha sido reducida a indicadores, variables, productos y resultados controlables, mesurables, perceptibles; ese sujeto del que lo humano es frecuentemente evadido (Ducoing, 2005: 164).

Ante esta situación se pretende que esta investigación parta de los sujetos en contextos situados de su práctica una vez que concluyen su proceso de formación. No pretende evaluar un programa de formación, intenta comprender los sentidos que los sujetos le atribuyen a su práctica desde sus valoraciones y concepciones particulares. ${ }^{5}$

${ }^{5}$ En este caso solo se consideran dos actores: los directivos y profesores; los primeros porque son los destinatarios directos del Programa de Formación (PFDC, 2016), los segundos porque pueden dar cuenta de los cambios que se dan a partir de que su director recibió la capacitación por parte de las instancias formadoras de docentes. 
Se entiende por sentidos la relación de atributos, valoraciones e ideas dentro de un contexto de significantes mediados por metarrelaciones asignados a una situación o experiencia; un ejemplo de sentido sería el atribuir a la experiencia de formación un mejor desempeño o una mayor capacidad en la toma de decisiones.

Ambos atributos: 1. Mejor desempeño, y 2. Mayor capacidad, son dos tipos de sentidos que los actores designan como parte de una experiencia propia o bien de un discurso que ellos quieren incorporar a un ethos de la profesión.

Desde esta mirada, es importante comprender los sentidos que los sujetos poseen una vez que concluyen su formación en relación con la experiencia de su gestión situada. En los apartados siguientes se intenta dar cuenta de este cometido.

\section{El concepto de formación, sus implicaciones en la vida profesional}

La formación es una vivencia que implica procesos de heteroformación, autoformación y coformación, entendidos como escenarios donde se aprende en contextos de pares que comparten saberes, conocimientos, habilidades, centrados en vivencias, experiencias, problemas, visiones y dilemas de la práctica profesional. Estos escenarios pueden ser los espacios formales e informales, presenciales o en redes, en comunidades que permiten desarrollar un ethos o código que orienta la acción propia del oficio. Yuren define el ethos como: "un sistema disposicional que se configura para resolver los problemas sociomorales que surgen en el campo de la profesión que se ejerce" (Yuren, 2007: 3)

Siguiendo esta lógica, el ethos es un elemento fundamental de la formación porque constituye un ámbito diferenciado de acción y reflexión de acuerdo a los problemas propios de la profesión, es un engranaje y motor de diversos elementos que configuran los procesos formativos (Yuren, 2007).

Los cursos de formación continua que los directivos toman en su trayecto a través de procesos de capacitación y actualización pueden ser formativos siempre y cuando los incorporen a sus saberes, los transfieran a su práctica cotidiana, a los estilos de resolución de conflictos, a las acciones que realizan en su hacer, a las actitudes y reflexiones que acompañan la práctica y que dan sentido propio al ejercicio de la gestión cotidiana.

La formación institucional que se ofrece a los docentes y directivos por parte del sistema educativo se concibe como inicial y continua. La formación inicial generalmente se adquiere en las escuelas normales y la formación continua se ofrece a través de cursos de actualización, de talleres breves de actualización y de posgrados profesionalizantes, ofrecidos tanto por las secretarías de educación de los estados del país, así como por instituciones de educación superior tanto públicas como privadas.

Desde esta dimensión, la formación continua es entendida como un conjunto de acciones intencionadas orientadas a la conformación de aprendizajes profesionales que se desarrollan durante 
el trayecto del desempeño con el objetivo de mejorar la práctica, de actualizar los conocimientos o de adquirir nuevas competencias orientadas a satisfacer las demandas educativas de los actores situados en una realidad educativa (Ferry, 1990: 52).

Un modelo de formación continua implica un proceso de adquisición sistemática de actitudes, conceptos, conocimientos, reglas o destrezas que se verán reflejados en la cotidianidad del profesional y que conllevan a transferencias de lo aprendido a situaciones concretas de la vida profesional, lo que también se verá reflejado en concepciones y creencias distintas sobre el ser y el hacer.

Como lo ha señalado Yurén (2007), la formación profesional no se puede circunscribir a procesos heteroformativos, como los que ofrecen los cursos formales e instituidos, sino a la autoformación y coformación que se da a través de procesos vivenciales e instituyentes implicados en la misma heteroformación; en este trabajo nos centraremos en el sentido que adquiere para los actores la formación proveniente de instancias oficiales, pero que toma nuevas significaciones en el espacio de intersubjetividad porque interesa valorar el sentido de la formación continua en el hacer y ser de los directivos que concluyen su proceso de actualización desde la opinión de los propios directivos y la comunidad escolar.

De manera concreta se aborda una variable consistente en destacar los sentidos de relevancia de los cursos en la práctica cotidiana de los propios directivos, esto es, si tienen algún sentido en su formación profesional y qué tanto son útiles en su práctica de gestión cotidiana. Esta variable se liga a la necesidad de concebir la formación desde un espacio dialógico y de interexperiencia, lo que constituye "el suelo, el centro de gravedad de la formación, cualquiera que sea su forma" (Honoré: 1980: 26), ya que desde la mirada de este autor la formación no implica un saber entendido como objeto que se pueda capitalizar, darse o imponerse, sino una comprensión, una relación del saber en situación.

De ahí la relevancia de la formación en el sentido de comprender los procesos de apropiación como una manera de reflexión propia desde lo aprendido, con nuevas significaciones a la acción cotidiana. No se trata de traducir la formación en una experiencia evaluable desde un dispositivo técnico que mida el logro en la práctica, sino de comprender los sentidos que apropia el sujeto y que incorpora a un ethos de su profesión (Yurén, 2007).

Por esta razón se considera importante, para los propósitos de este estudio, centrar la mirada en los sujetos desde sus formas de subjetivación, esto es, de maneras de interpelar la formación desde la acción. No pretende evaluar un programa, ni tomar como categorías analíticas los propósitos de un programa, porque eso implicaría ir a la realidad de los sujetos con un prejuicio fundado en la verificabilidad de indicadores prescritos de antemano. Por el contrario, se pretende desde el imperativo fenomenológico comprender el fenómeno desde el actor.

La recuperación de los sentidos se infirió de las respuestas que nos ofrecieron los sujetos, a quienes se les aplicó una entrevista de cuyas preguntas se rescataron tres para este análisis: ¿Con- 
sidera que la tarea del directivo se puede realizar sin necesidad de tomar este curso ${ }^{6}$ ¿Cree que el curso es suficiente para que un director tenga una mejor gestión? ¿Existe diferencia entre lo que se aprende en el curso y la realidad de su escuela?

En el análisis se integra una interpretación de los sentidos inferidos de sus respuestas, que permiten comprender las implicaciones del dispositivo de formación.

La necesidad de comprender los sentidos de los directores que han concluido su proceso formativo nos lleva a mirar no sólo a la experiencia que dicen les ha dejado el proceso de actualización, sino a comprender "las estructuras profundas" (Flick, 2007) que le dan sentido a sus opiniones al estar investidas de significados. La metodología consistió en la aplicación de entrevistas estructuradas a 23 directivos y 92 docentes de 23 escuelas primarias ubicadas en seis regiones del estado de Jalisco, las cuales fueron seleccionadas dada la concentración de directores capacitados en el curso mencionado (PFDC, 2006). Estas seis regiones son las siguiente: Altos Norte (AN), Altos Sur (AS), Centro (C), Costa Norte (CN), Costa Sur (CS) y La Ciénega (LC). Se eligieron las escuelas bajo el procedimiento del muestreo aleatorio simple. A continuación, se muestra el cuadro de las escuelas, directivos y docentes entrevistados:

\section{Cuadro 1. Directores y profesores entrevistados}

\begin{tabular}{|c|c|c|c|}
\hline Códigos $^{7}$ & Profesores & Directores & Regiones \\
\hline AN/LM/JM & 3 & 1 & Altos Norte \\
\hline ANOANH & 5 & & A tos Norte \\
\hline $\begin{array}{l}\text { AS/CIRPP } \\
\text { AS/CGR }\end{array}$ & $\frac{4}{5}$ & & $\frac{\text { Altos Sur }}{\text { Altos Sur }}$ \\
\hline C/GD/AY & & & Costa Norte \\
\hline C/GD/OR & $\frac{6}{2}$ & & Costa Norte \\
\hline C/GD/CE & & & $\begin{array}{l}\text { Costa Sur } \\
\text { Costa Sur }\end{array}$ \\
\hline C/GD/SR & & & Costa Sur \\
\hline C/GD/EZ & & & La Cienega \\
\hline C/TL/EP & 4 & & La Cienega \\
\hline C/GD/IR & & & La Cienega \\
\hline C/TL/CO & & & Centro \\
\hline C/GD/SA & & & Centro \\
\hline LCOOCIAN & 3 & & Centro \\
\hline 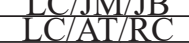 & & & $\begin{array}{l}\text { Centro } \\
\text { Centro }\end{array}$ \\
\hline CN/PV/15M & & & Centro \\
\hline $\begin{array}{l}\text { CN/PV/BJ } \\
\mathrm{CS} / \mathrm{CC} / \mathrm{PT}\end{array}$ & & & $\begin{array}{l}\text { Centro } \\
\text { Centro }\end{array}$ \\
\hline CS/C/MLL & 3 & & Centro \\
\hline CS/TC/MD & 6 & & Centro \\
\hline 23 escuelas & 92 docentes & 23 directores & 6 region \\
\hline
\end{tabular}

Fuente: elaboración propia

${ }^{6}$ El curso referido en el caso de este estudio es el Programa de Formación de Directivos por Competencias 2006 (PFDC, 2006), el cual no tuvo como objetivo evaluar el curso en sí mismo, sino rescatar los sentidos que tuvo el curso para los directivos que lo tomaron.

${ }^{7}$ La nomenclatura de la codificación se construyó como criterio de identificación de acuerdo con el siguiente orden: iniciales de la región/iniciales del municipio, iniciales del nombre de la escuela. Ejemplo: región Norte Sur-NS. Municipio: Puerto Vallarta: PV. Escuela Amado Nervo: AN, siendo así, el código de esta referencia sería: NS/PV/AN. En el caso de las entrevistas se aporta el dato e, si es diario de campo, se agrega a letra d. 
La presentación de los resultados del análisis pondera respuestas de las cuales se infirieron sentidos comprendidos como atributos asignados desde la valoración de los sujetos. A continuación se analizan cada uno de los sentidos inferidos de dichas respuestas.

\section{Los cursos proveen de certidumbre en la práctica}

En este apartado se concentran las perspectivas de los directivos inferidos a partir de las respuestas que ofrecieron:

a) El curso nos ofreció mayores conocimientos acerca de la gestión, lo que nos permitió conocer y tomar decisiones sobre asuntos relacionados con la gestión.

b) Los temas abordados en el curso no se habían considerado o analizado, ni habíamos tenido la oportunidad de revisar y valorar sus implicaciones.

c) La participación de los formadores fue importante.

d) Los veintitrés afirman que el curso resultó de utilidad.

El sentido de utilidad es ponderado de acuerdo a valoraciones, ya que nueve directores ponen énfasis en el aprendizaje de propuestas para la intervención y la mejora en el campo de la dirección escolar; expresan que el curso fue bueno en el sentido de que ofreció conocimientos teóricos para orientar sus acciones y a la vez les permitió un mayor acercamiento a propuestas para la mejora de su práctica y el manejo de reglamentos y normatividades para intervenir y resolver conflictos a los que algunos de ellos ya se enfrentaban, como se podrá evidenciar en su propia narración:

1. Es esencial llevar la práctica con la teoría y nos sirvió el curso, con la teoría fundamental porque a veces creemos que estamos haciendo las cosas bien, y es necesaria la profesionalización del directivo que es el eje motor de la escuela y para mi si es importante cursar el diplomado (AN/O/NH/d).

2. Se podría ser directivo sin diplomado, pero éste sí ayuda a mejorar las prácticas porque dejamos de actuar por ensayo y error. Por ejemplo, para que se dieran escuelas de calidad pasarían diez años, con el diplomado se lograría en cuatro, tres o cinco años. Sobre todo, que se manejan algunas cuestiones administrativas y legales que desconocemos y que sí es necesario saber porque facilitan procesos (C/GD/MLM/d).

3. Yo creo que sí necesitamos el curso, yo aprendí muchas cosas y mire aquí están mis materiales en los que me apoyo para consultar algunas cosas. Son materiales muy valiosos, yo creo que eso fue lo mejor del diplomado, las lecturas, porque las podemos consultar (C/GD/SA/d).

4. Creo que el diplomado fue de gran utilidad, es un diplomado joven, yo nunca lo había escuchado, yo me inscribí a éste de manera voluntaria y creo que el 
diplomado es conveniente que lo tomemos todos los que asumimos el cargo. Qué bueno que existe esa preocupación por parte de la Secretaría y para mí sí fue necesario y tomaría de nuevo una segunda parte (LC/OC/AN/d).

5. A partir del diplomado he tenido más armas para trabajar con mi inspector, el inspector ha venido a traernos proyectos y ya con la experiencia del diplomado me he sentido seguro de decirle que algunos de sus proyectos están mal que tiene errores en su diagnóstico, que se pueden mejorar algunos aspectos.

6. El diplomado te enseña muchas cosas, como ser ordenado, como llevar planes de trabajo, hubo muchos contenidos que hasta el momento me han servido, como la planeación y contenidos que he compartido con mis compañeros docentes. En el diplomado nos enseñaron que antes de cualquier proyecto debíamos hacer un diagnóstico y es algo que no me había puesto a pensar. El diplomado con el contenido, nos ha dado armas y herramientas para un mejor trabajo (CN/PV/BJ/d).

7. El programa de directivos es bastante bueno ya que nos lleva a conocer más a fondo la situación de un proceso de trabajo, como empresa, como trabajo y como padre de familia (CS/C/MLL/d).

8. Este diplomado fue el mejor que he recibido, por su diseño, sobre todo el diseño de instrumentos para dar seguimiento y evaluación que es la parte más flaca de toda la cuestión pedagógica, hacemos planes y programas y van al cajón, pero nunca hay un seguimiento, pero ya este programa como que hizo mucho hincapié en elaborar esos instrumentos para dar tu seguimiento a " $x$ " plan que laborabas $o$, trabajo que planteabas, yo elaboré un instrumento muy sencillo que me permite ver lo que se ha hecho aunque incomode de alguna manera al personal, pero es lo que nos permite ver los avances, y que el mismo equipo docente sea el constructor de sus planes sin esperar gente externa porque estaría uno muy dependiente siempre como ha sido ¿No? que me digan cómo hacer esto, no, hay que hacerlo uno, hay directores que no han ido al programa todavía, la están haciendo pero no creo que en las mismas condiciones que nosotros, ni con la misma calidad (CS/TC/MD/d).

9. ... yo se lo recomendé a varios compañeros que no lo conocían para que fueran y lo tomaran porque sí hay algunas propuestas bastante buenas, para que un director pueda mejorar en su escuela, yo aquí antes tenía muchos conflictos a veces, era yo misma quien me obstaculizaba, porque no tenía nada organizado (AS/TP/RP/d).

Trece de los directores entrevistados valoran el curso en cuanto a que éste les dio elementos para tener mayor seguridad en su desempeño como directores nuevos o con pocos años de haber adquirido el cargo; ante ello reconocen que su capacitación les dejó experiencias nuevas que podrán incorporar a su práctica, como podemos ver en las siguientes viñetas narrativas: 
1. El diplomado sí fue un gran apoyo y me ayudó a desempeñarme con seguridad $(\mathrm{C} /$ $\mathrm{GD} / \mathrm{AY} / \mathrm{d})$.

2. Considero que el discurso es muy valioso porque nos deja mucha experiencia $(\mathrm{C} /$ $\mathrm{GD} / \mathrm{AO} / \mathrm{d})$.

3. Cuando uno es recién egresado de maestro frente al grupo y pasa a dirección sí se necesita un diplomado para encausar las perspectivas y la gestión, todo lo que se desconoce $(\mathrm{CGD} / \mathrm{CL} / \mathrm{d})$.

4. ...muchos directivos nuevos fueron maestros de grupo y como maestro de grupo se desconocen muchas estrategias para la gestión, pero este diplomado ofrece herramientas que permiten mejorar nuestra gestión $(\mathrm{AS} / \mathrm{CP} / \mathrm{RP} / \mathrm{d})$.

5. El programa te abre un portal de formas para trabajar que muchas veces no conocemos, ya que necesitamos orientación y tener todo en orden conforme al derecho $(\mathrm{C} / \mathrm{GD} / \mathrm{IG} / \mathrm{d})$.

6. Este curso que yo lleve, claro es muy pesado, es muy práctico pero es demasiado útil y yo considero que no debería darse el puesto de director a quien no haya tomado ese curso porque es esencial, si bien nosotros por la misma práctica de que hemos [estado] obligados a hacer muchas cosas a través de la experiencia que las fuimos aprendiendo y aplicando, no hay como el preparase y este curso que tomamos nosotros, pues es importantísimo, de que es pesado porque es práctico y teórico, aunque es más práctico, a lo que me refiero es que hay que estar trabajando para desarrollar el curso, sí, ese es el detalle (C/GD/EZ/d).

7. Se realiza mejor con conocimiento, tomando el diplomado se adquiere mayor conocimiento por lo tanto más herramientas con las cuales poder trabajar $(\mathrm{C} / \mathrm{GD} /$ $\mathrm{SR} / \mathrm{d})$.

8. La capacitación nos da beneficios, a partir del curso yo me siento más segura con lo que estoy haciendo y considero que sí me sirvió muchísimo (C/TL/EP/d).

9. Yo creo que es muy importante este diplomado porque cuando uno gana la plaza de director ya sea por oposición o algún examen, muchas veces el maestro brinca de maestro a director, y uno muchas veces no sabe lo que se trabaja en la dirección, entonces todas las cosas importantes, sobre todo la normatividad, muchas veces el maestro de grupo no lo toma en cuenta, entonces muchas veces es importante para darle el manejo así, falta darle esa capacitación, que el directivo sepa que es lo qué debe de hacer en cada uno de los rubros (C/GD/IR/d).

10. Me gustaría que se le diera seguimiento, aunque es excelente aún quedan muchas dudas y pues debemos seguir actualizándonos y preparándonos, yo considero que se requiere más seguimiento, más acompañamiento, siempre hay situaciones que nos generan dudas y solamente si seguimos actualizándonos y preparándonos 
podemos hacer mejor nuestro trabajo, porque todo evoluciona, los niños, los padres, la sociedad (C/GD/CO/d).

11. Este curso me dio más seguridad y aprendí muchas cosas. Me siento más segura de lo que hago y digo. Tuve muchos conocimientos nuevos (CN/PV/15M/d).

12. Muchos compañeros reconocieron que lo que les dio el diplomado fue muy importante y dijeron: "Si nos hubieran dicho esto con tiempo nos hubiéramos ahorrado muchos problemas" (LC/JM/JB/d).

13. Yo ya estaba pidiendo algo así desde hace tiempo, aunque fuera una guía de normatividades, reglas, apoyo para lo técnico pedagógico. Aunque este curso es nuevo y a muchos lo nuevo les provoca temor, pues debemos de tener disposición, porque son herramientas para trabajar mejor. Yo sí esperaba que nos capacitaran y el curso me pareció bueno, aunque fue acelerado porque se necesitaba más tiempo (LC/AT/RC/d).

En términos generales, los directores entrevistados consideran que el curso fue un elemento importante en su formación, aunque no suficiente ya que señalan otras situaciones que deben completar la capacitación como serían: la vocación y la inclinación hacia la función directiva, el seguimiento, la tutoría, ofrecer más tiempo para las actividades, entre otros aspectos.

Al respecto ellos señalan situaciones que deberían implementarse en el curso para que éste tuviera mayor impacto en las prácticas, y que a continuación señalaremos.

\section{Se demanda mayor acompañamiento tutorial}

Los directivos reconocen que una de las fortalezas más importantes del curso fue precisamente el acompañamiento encarnado en la figura del tutor. En muchos de los casos, esta fortaleza sólo se dio al inicio en seis visitas en promedio, pero los directivos consideran que el acompañamiento tutorial que incluye el PFDC (2006), y que se entiende como una fase de formación situada incluida en el propio proceso de formación, debe ampliar su radio de acción y ser un ejercicio permanente, ya que el tutor representa una presencia formativa y horizontal, aunque con mayor experiencia, conocimientos y una actitud cooperativa hacia la mejora, que permite la reflexión, el análisis, la autoevaluación in situ, ya que éste: recomienda, sugiere, amplía perspectivas, problematiza y ofrece alternativas de solución en algunos casos.

La valoración que los directivos hacen de 22 tutores fue positiva, reconociendo en esta figura un apoyo y un aprendizaje en la formación; sólo en uno de los casos se reportaron comentarios insatisfactorios del tutor por parte de un directivo, quien lo vio como una figura amenazante e intimidatoria. 

escuelas primarias

\section{Las evidencias de la formación que reconoce la comunidad escolar}

Del universo de 92 profesores de las 23 escuelas, ochenta reconocieron que a partir de que su director tomó el curso por competencias éste ha procurado un mayor número de reuniones y de trabajo colegiado.

El resto, constituido por 12 profesores, señaló que las reuniones que se organizan son las ya establecidas por el calendario y la agenda escolar.

Ahora bien, los profesores valoraron que, si bien hay más reuniones de trabajo colegiado, en estos encuentros a veces no se dan acuerdos y en algunos casos reportan que los acuerdos tomados ya no tienen seguimiento porque el director toma otras decisiones y deja de lado dichos acuerdos. Ante esto cabe reflexionar sobre las condiciones propias de cada contexto, en el sentido de que la rigurosidad en el manejo de los tiempos sigue dependiendo, en la mayoría de las escuelas, de la hegemonía de una agenda centralizada en la figura de la autoridad externa y que por ello se vuelve regulador de la administración escolar.

De acuerdo con las apreciaciones de los docentes y directivos podemos considerar que el modelo administrativo centrado en la escuela aún vive las tensiones generadas por las tendencias externo reguladoras provenientes de la organización de la secretaría de educación, concretada en la figura de la supervisión escolar.

En este sentido, desde la mirada de los directivos, la supervisión resulta una instancia ajena a las demandas y prioridades escolares; ante ello los directores demandan un diálogo entre ambas agendas y un mayor acercamiento de la supervisión en asuntos de índole escolar y no sólo de administración burocrática, centralizada en las instancias externas.

\section{Las carencias que los actores reportan desde su formación}

La mejor manera de evaluar los efectos de la formación es la valoración de lo que los sujetos logran en la propia práctica, puesto que ahí se palpan con mayor claridad los logros y, fundamentalmente, los problemas para aplicar lo que se aprende en los espacios formales de la capacitación y con base en ello generar propuestas para mejorar dichos procesos de formación. Por ello, las dificultades para aplicar las competencias en las escuelas son evidenciadas desde la práctica de los directivos y son las siguientes:

a) La disociación teoría y práctica, un conflicto latente en la formación

Hay un problema interesante que los directivos reportan y que tiene que ver, como ellos mismos lo revelan, con aspectos de la tradición formativa, que es la disociación y asimetría entre la teoría y la práctica. Así, ellos revelan en su entrevista aspectos como:

- El curso en algunos momentos fue muy teórico y la teoría no era aplicable a los contextos de las escuelas.

- Hubo un exceso de ejercicios que no eran aplicables a la realidad. 
- No partía de situaciones que ocurren realmente en la gestión cotidiana, por tanto, había disociación entre la realidad vivida en las escuelas y los ejercicios que nos propuso el curso.

Aunque el propósito no es analizar la naturaleza o la génesis de estas problemáticas, sí surge la necesidad de abordar la problematicidad de la tradición formativa basada en la concepción de que el conocimiento se debe aplicar en la realidad, pero sin una claridad de las mediaciones necesarias entre la práctica y la teoría; en algunos casos la noción mediacional se aproxima a una concepción mecánica y lineal basada en el ideal de que lo aprendido se tiene que aplicar. El modelo de formación dentro de esta experiencia carece de elementos mediacionales e integradores entre lo aprendido y lo vivido; y, finalmente, le apuesta al voluntarismo y a la pericia del sujeto en situación. El mismo programa de formación de directivos refiere una modalidad en alternancia en el sentido siguiente:

La modalidad que se propone para la formación del director de escuela de Educación Básica se basa en la alternancia, esto es, la sucesión de actividades teóricas y prácticas. Se conforma como un esquema a través del cual el director recibe información e inducción teórica, los cuales pondrá en práctica. Esta aplicación de la teoría en su práctica, le permitirá aprender, continuar o redefinir la forma en que conducirá su escuela (Esquivel et al., 2011: 179). ${ }^{8}$

Como podemos ver, el mismo modelo favorece una formación basada en la adquisición de conocimientos, porque, como lo señala la cita anterior, el director recibe información teórica que luego pondrá en práctica; pero no refiere un factor mediacional entre ambas dimensiones y se aleja de un modelo situacional que postula que:

quien se forma emprende y prosigue, a lo largo de su carrera, un trabajo sobre sí mismo, que consiste en la desestructuración y reestructuración del conocimiento de su realidad, con lo cual concibe proyectos de acción adaptados a su contexto y a sus propias posibilidades. Implica, por tanto, investir su práctica y formarse (Ferry, 1997: 140).

Sin embargo, es necesario reconocer a partir de algunas reflexiones que los mismos directivos hacen, que la relación entre la teoría aprendida y la vivencia práctica no puede ser mecánica ni simple, puesto que involucra, por una parte, al sujeto en formación en su plena totalidad: desde sus valores, actitudes, relaciones, emociones, afectos, rechazos, identidades, rupturas, dificultades o conflictos; por otra parte, también involucra las condiciones de los contextos de aplicación en la práctica.

${ }^{8}$ El subrayado es propio. 
La relación entre teoría-práctica tiene que ver con procesos de apropiación, más que de interiorización. La apropiación se conforma desde situaciones dialógicas e intersubjetivas involucrando un nivel de adquisición de conciencia práctica. Lo segundo, la interiorización, implica el nivel que cada sujeto logra al incorporar el saber a un esquema cognitivo. Por lo cual, el concepto de apropiación nos permite ir más allá de la noción de interiorización, y con ello destacar el proceso por el cual los directores participan en tanto agentes en contextos concretos.

Este supuesto lo podemos constatar con las respuestas que nos dieron a la pregunta: ¿Existe mucha diferencia entre lo que se aprende en el diplomado y la realidad de la escuela donde trabaja? Las respuestas ofrecidas por parte de los directivos se pudieron clasificar en tres niveles, los que consideran que sí hay diferencias entre lo aprendido y la realidad, por ello se requiere hacer una adaptación entre ambas dimensiones; quienes consideraron que sí hay diferencias entre ambas, pero que existen cosas que no son aplicables; y finalmente quienes consideraron que no hay diferencia entre ambas, por tanto todo es aplicable.

Obviamente los tres tipos de respuesta no se pueden clasificar de manera tajante o maniquea puesto que revelan la dinámica del directivo al concretar lo aprendido al ámbito de aplicación de sus competencias. En ese sentido, los puntos de vista reflejan el juicio prudencial ${ }^{9}$ del directivo de acuerdo con los contextos de aplicación y los sentidos que el propio sujeto le da al saber frente a la realidad, a los problemas y a la comunidad, como podemos verlo en el siguiente cuadro de análisis que se desprende de la pregunta: ¿existe diferencia entre lo que se aprende en el curso y la realidad de su escuela?

${ }^{9}$ Le llamamos juicio prudencial al juicio en situación; a aquel que se asume cuando frente a la dimensión normativa del sujeto se presenta una situación problemática donde él tiene que asumir una actitud deliberativa frente a las posibles consecuencias de su actuación. Los profesionales no siempre actúan con base en un código deontológico del deber ser, sino ante la reflexión de lo que puedo hacer y las consecuencias propias de mi hacer. Yuren (2007) también le llama a este juicio eticidad crítica. 
Cuadro 2. Diferencias entre lo aprendido y la realidad escolar

\begin{tabular}{|c|c|c|}
\hline $\begin{array}{c}\text { Sí hay diferencias. Por eso hay que } \\
\text { adaptar lo aprendido al contexto } \\
\text { escolar }\end{array}$ & $\begin{array}{l}\text { No hay diferencias entre lo } \\
\text { aprendido y la realidad. Todo lo } \\
\text { aprendido es aplicable }\end{array}$ & $\begin{array}{c}\text { Sí hay diferencias. No todo es } \\
\text { aplicable }\end{array}$ \\
\hline $\begin{array}{l}\text { Cada contexto es diferente, y el } \\
\text { diplomado, bueno tiene sus diferentes } \\
\text { ejemplos, nos da unas bases, pero las } \\
\text { tienes que adaptar a tu contexto (C/ } \\
\text { GD/SR/d). }\end{array}$ & $\begin{array}{l}\text { No, porque son herramientas que nos } \\
\text { ayudan en la gestión diaria, y por eso } \\
\text { reitero que debería de ser obligatorio } \\
\text { que los directores tomen ese curso, } \\
\text { son herramientas que nos dan (C/TL/ } \\
\text { EP/d). }\end{array}$ & $\begin{array}{l}\text { Pienso que sí. Cada escuela tiene } \\
\text { problemas y contextos diferentes. Sí } \\
\text { hay diferencias. Porque una cosa es la } \\
\text { teoría y otra la práctica }(\mathrm{C} / \mathrm{GD} / \mathrm{AY} / \mathrm{d}) \text {. }\end{array}$ \\
\hline $\begin{array}{l}\text { Sí, aunque hay que hacer adaptaciones } \\
\text { de acuerdo a nuestro contexto (C/GD/ } \\
\text { OR/d). }\end{array}$ & $\begin{array}{l}\text { No, puesto que todas las herramientas } \\
\text { que nos dieron o que nos proporcionó } \\
\text { el programa pueden ser retomadas } \\
\text { para resolver situaciones en la } \\
\text { escuela, no hay mucha diferencia con } \\
\text { lo que aprendimos, pero debemos de } \\
\text { conjuntar la teoría con la práctica, si } \\
\text { no estamos acostumbrados a buscar en } \\
\text { libros o en leyes las respuestas, pues } \\
\text { no es fácil (LC/OC/AN/d). }\end{array}$ & $\begin{array}{l}\text { Sí existe, hay diferencia entre lo } \\
\text { teórico y lo práctico, pero nos ayudó } \\
\text { mucho en la normatividad, para } \\
\text { conocerla y saber cómo actuar en } \\
\text { algunos momentos. Se nos presentan } \\
\text { muchos casos fuera de lo cotidiano, } \\
\text { que nunca se analizaron, pero } \\
\text { conocer la normatividad nos ayudó } \\
\text { mucho para saber cómo actuar en } \\
\text { algunos momentos y sobre todo cómo } \\
\text { organizarnos mejor todo el personal } \\
\text { junto. }\end{array}$ \\
\hline $\begin{array}{l}\text { Sí existe alguna, no mucha, pero } \\
\text { alguna, ya que el diplomado está } \\
\text { pensado en una forma general y } \\
\text { basada sobre las leyes y reglamentos } \\
\text { que rigen a la Secretaría de Educación, } \\
\text { pero el entorno donde se realiza la } \\
\text { actividad educativa no es el mismo en } \\
\text { todas las escuelas, las comunidades } \\
\text { son distintas y las necesidades son } \\
\text { diferentes }(\mathrm{C} / \mathrm{Z} / \mathrm{IG} / \mathrm{d}) \text {. }\end{array}$ & & $\begin{array}{l}\text { El diplomado en su estructura y } \\
\text { organización tiene cosas de más y } \\
\text { ausencias. Por ejemplo, en las visitas } \\
\text { de tutores hace falta tiempo para hacer } \\
\text { las cosas que sugiere el diplomado, a } \\
\text { falta de esos tiempos se hacen muchas } \\
\text { cosas de manera hipotética, entonces } \\
\text { en la estructura hay cosas como los } \\
\text { diagramas que nunca vamos a usar } \\
\text { y donde debemos hacer cosas que } \\
\text { realmente nos están pasando y no } \\
\text { de manera hipotética y ficticia. En } \\
\text { ese sentido el diplomado está muy } \\
\text { alejado de la realidad porque se parte } \\
\text { de situaciones hipotéticas y no de } \\
\text { problemas reales }(C / G D / M L M / d) \text {. }\end{array}$ \\
\hline $\begin{array}{l}\text { Sí hay muchas diferencias, que cada } \\
\text { director debe adaptar a su realidad, } \\
\text { porque cada contexto es distinto (C/ } \\
\mathrm{GD} / \mathrm{CO} / \mathrm{d}) \text {. }\end{array}$ & & $\begin{array}{l}\text { Creo que hay cosas que no se pueden } \\
\text { aplicar, en parte, el diplomado nos } \\
\text { sugiere tener todo organizado, pero } \\
\text { la realidad es muy distinta a lo que se } \\
\text { ve en un curso, no es difícil, pero la } \\
\text { realidad nos impone otras cosas (LC/ } \\
\mathrm{JM} / \mathrm{JB} / \mathrm{d} \text { ). }\end{array}$ \\
\hline
\end{tabular}

Fuente: elaboración propia 


\section{b) La ausencia del director en las escuelas durante su formación}

Los veintitrés directivos coinciden en que ausentarse dos días de la semana durante cierto lapso para tomar las sesiones presenciales que les demanda el curso es una situación complicada, ya que acarrea tensiones y en ocasiones conflictos en sus comunidades escolares.

Estos conflictos tienen que ver con el papel de la presencia física en la escuela que tanto docentes como padres de familia esperan tener del directivo, su ausencia se traduce en una opinión poco favorable hacia el directivo de parte de docentes y padres de familia; como lo narran los directivos a la pregunta: ¿Los profesores saben que usted está tomando el diplomado por competencias?

Sí, ellos saben; bueno, sólo los nuevos que acaban de llegar no lo saben. Eso lo aclaré desde un principio, sabiendo que iba a haber problemáticas, porque al estar ausente se dieron muchos problemas en la escuela que requirieron mi presencia $(\mathrm{C} / \mathrm{GD} / \mathrm{OR} / \mathrm{d})$.

Sí, yo les advertí dado que tenía que faltar dos veces a la semana, asunto que se me hace muy complicado, para mí bastaría ir al curso una vez a la semana. Los maestros se dieron cuenta a partir de las ausencias (C/ GD/MLM/d).

En el registro también se constató esta percepción de la propia directora con respecto al tiempo de capacitación presencial que le demando ausentarse algunos días de la escuela:

En la visita la directora refiere el problema de tener que ausentarse de la escuela para cubrir los requerimientos de su capacitación, porque esto, a decir de ella, es muy problemático con los padres de familia, ya que los padres de familia al no ver a la directora en la escuela juzgaban que era una directora faltista. Ella sugiere que sean turnos o tiempos que no interfieran con su presencia en la escuela. También que la Secretaría haga extensiva la información a los padres para que haya un apoyo mayor de la institución o bien cómo hacer horarios para que no se interfiera con horas clases (C/GD/OR/r/17/06/2011).

Ante esta problemática sentida por la comunidad escolar, los directores proponen una capacitación a contraturno o bien un mayor apoyo de parte de la Secretaría de Educación, ya que consideran que, así como se envía la convocatoria, de la misma manera se comunique a la comunidad escolar sobre la justificación y el motivo de las ausencias del directivo a su plantel.

Es importante analizar cómo un aspecto intrínseco a un factor de planeación del curso, que para algunos podría ser irrelevante, sí tiene implicaciones en la práctica de la gestión y en la evaluación del propio curso por parte de los directivos, ya que los directores que presentaron esta problemática refirieron que cuando se presentaban a sus escuelas después de las ausencias tenían largas listas de pendientes en sus escuelas, problemas no resueltos que esperaban la presencia del directivo y algunas inconformidades de parte de los padres de familia. 
Después de sopesar las opiniones y percepciones en los actores concluimos que, en efecto, la organización de los tiempos de la capacitación es un elemento que puede considerarse para la mejora del programa mismo y sobre todo de sus efectos en las prácticas de la gestión de los directivos escolares.

c) Una ausencia de miradas integradoras en los saberes de la gestión

Este punto, que consideramos de suma importancia por las implicaciones en la aplicación de las competencias, es el referido a la mirada integradora de los saberes de la gestión y tiene que ver propiamente con la pericia, la sensibilidad y el compromiso del directivo ante los problemas que demandan su intervención. A manera de supuesto es notorio el problema de integración del saber conceptual y el procedimental ante situaciones de la gestión que demandan una integración de saberes.

\section{Algunas conclusiones}

Indudablemente los cursos formativos ofrecen, desde los sentidos de los directivos entrevistados, mayor certidumbre y un sentido de reconocimiento en la práctica.

Sin embargo, los entrevistados refieren que en su formación es importante partir de situaciones más apegadas a las problemáticas escolares; trabajar estudios de caso y situaciones que se puedan transferir a la práctica y les permita visualizar modelos que viabilicen la transferencia del saber.

Uno de los problemas que refieren en la formación es la asimetría entre la teoría y la práctica. Situación que podría resolverse si se enfatizaran experiencias prácticas donde la transferencia del saber fuera concretada, valorada y asumida por los directivos en formación.

Dados los escenarios de incertidumbre de las escuelas, un modelo de formación tendría que satisfacer las posibilidades de transformación desde contextos reales y por tanto impredecibles, como lo señala Loya al considerar que la formación situacional:

Es un proceso al cual se expone el formante al enfrentar la singularidad de las situaciones en las que se ve implicado como educador, de tal manera que los efectos formadores son parciales e inesperados porque surgen de la relación que establece el sujeto con la realidad y no de alguna programación preestablecida y controlable. La formación se fundamenta en lo imprevisible y lo no dominable (Loya, 2008: 5).

Esta demanda está presente en la voz de los directivos, quienes proponen mayor énfasis en las prácticas y partir del contexto situado en las escuelas.

Lo anterior tiene que ver con un asunto relevante y necesario para todo proceso de formación: cuando se forma a un sujeto también se forma a una comunidad, pues como señala Honoré (1980), toda formación implica una interexperiencia comprensiva con el espacio vital del actor en situación. 
¿Son formativos los cursos de capacitación continua? La perspectiva de los directores y docentes de escuelas primarias

Así se constata cuando a la pregunta dirigida a los docentes de las escuelas sobre si consideran que su directivo se debe seguir actualizándose, sus respuestas coinciden en que no sólo el director debe actualizarse y seguirse formando, sino también ellos, los docentes; éstos consideran que la formación es un aspecto necesario, imprescindible e ineludible ante los cambios vertiginosos de la sociedad y la demanda de los sectores por una mayor calidad en y desde las escuelas, donde el supervisor escolar no puede quedar excluido.

Se infiere, por tanto, que la formación en la gestión no sólo corresponde al director sino a la comunidad docente y al supervisor, ya que las implicaciones de la formación obligan a resignificar las prácticas del contexto y demandan la movilización de las inercias por parte de quienes comparten su entorno. Este es un elemento referido por los directivos y la propia comunidad docente, quienes demandan procesos de acompañamiento y de formación a través de comunidades intersubjetivas que compartan experiencias en situación más que reclutamientos en espacios formales, donde los aprendizajes deben tener relevancia en la práctica de la gestión cotidiana.

\section{Referencias}

Acosta y Braslavsky (2001). El estado de la enseñanza de la formación en gestión y política educativa en América Latina. Buenos Aires: IIPE-UNESCO.

Aguilar, C. y S. Schmelkes (2001). "El estado de la enseñanza de la formación en gestión y política educativa en México”. En: Acosta y Braslavsky (2001). El estado de la enseñanza de la formación en gestión y política educativa en América Latina. Buenos Aires: IIPE-UNESCO.

Anzaldúa, A.R.E (2009) La formación, una mirada desde el sujeto. Ponencia X Congreso Nacional de Investigación Educativa. Área 15 procesos de formación. Veracruz, México.

Esquivel, M. et al. (2011). "El Programa de Formación de Directivos por Competencias". En: V. Ponce. Nuevos estilos de gestión de políticas educativas. Comunidades y redes de la Secretaría de Educación Jalisco. Guadalajara: SEJ.

Deleuze, G. (1999). “¿Qué es un dispositivo?”. En: Balbier et al. Michel Foucault, filósofo. Barcelona: Gedisa.

Ducoing, P. (Coord.). (2005). Sujetos, actores y procesos de formación, México: COMIE.

Ferry, G. (1991). El trayecto de la formación. México: Paidós.

(1997). Pedagogía de la formación. Serie Los Documentos 6. Buenos Aires: Novedades Educativas-UBA.

Honoré, B. (1980). Para una teoría de la formación. Madrid: Narcea.

Loya, H. (2008). "Los modelos pedagógicos en la formación de profesores". En: Revista Iberoamericana de Educación, núm. 46/3, 25 de mayo. Organización de Estados Iberoamericanos para la Educación, la Ciencia y la Cultura (OEI). 
Secretaría de Educación Jalisco (2006). Programa de Formación de Directivos por Competencias. Guadalajara: Secretaría de Educación Jalisco.

Schmelkes, S. (1998). "La educación básica". En Latapí Pablo. Un siglo de educación en México. Vol. I. México: CONACULTA/FCE.

Yurén, T. y C. Romero (2007). "Ethos profesional, dispositivo universitario y coformación". En: Revista Reencuentro. Análisis de problemas universitarios, núm. 49. Valores en las profesiones universitarias. México: Universidad Autónoma Metropolitana-Xochimilco, pp. 22-29.

Zorrilla, M. y M. G. Pérez (2006) Los directores escolares frente al dilema de las reformas educativas en el caso de México. Vol. 4. México: REICE.

Recibido: 30/09/15

Dictaminado: 19/04/16

Corregido: 20/04/16

Aceptado: 02/05/16 